\title{
Modulation of the cancer cell transcriptome by culture media formulations and cell density
}

\author{
SEUNG WOOK KIM, SUN-JIN KIM, ROBERT R. LANGLEY and ISAIAH J. FIDLER \\ Department of Cancer Biology, Metastasis Research Laboratory, The University \\ of Texas MD Anderson Cancer Center, Houston, TX 77030, USA
}

Received September 8, 2014; Accepted October 13, 2014

DOI: 10.3892/ijo.2015.2930

\begin{abstract}
We investigated how varying the composition of cell culture formulations and growing cancer cells at different densities might affect tumor cell genotype. Specifically, we compared gene expression profiles generated by human MDA-MB-231 breast cancer cells cultured in different media [minimum essential medium (MEM), Dulbecco's modified Eagle's medium (DMEM), or Roswell Park Memorial Institute (RPMI)-1640 medium] containing different concentrations of fetal bovine serum (FBS) or different sera (equine or bovine) that were grown at different cell densities. More than 2,000 genes were differentially modulated by at least a 2-fold difference when MDA-MB-231 cancer cells were $90 \%$ confluent and compared with cultures that were $50 \%$ confluent. Altering the concentration of serum produced an even more pronounced effect on MDA-MB-231 cancer cell gene expression in that 2,981 genes were differentially expressed in a comparison between cells cultured in $0.1 \%$ FBS and same cell density cultures that were maintained in $10 \%$ FBS. A comparison between MDA-MB-231 cancer cells that were $90 \%$ confluent in MEM, DMEM, or RPMI-1640 media, all containing $10 \%$ FBS, resulted in 8,925 differentially expressed genes. Moreover, one-quarter (25.6\%) of genes from our genome-wide expression analysis were expressed at significantly different levels by cells grown in MEM, DMEM, or RPMI-1640 media. Genes associated with epithelial-mesenchymal transition (EMT) were among the genes that were differentially modulated by cells grown in different cell culture formulations and these genes were verified at the protein level. Collectively, these results underscore the importance of accurate reporting and maintenance of uniform culture conditions to ensure reproducible results.
\end{abstract}

Correspondence to: Dr Isaiah J. Fidler, Department of Cancer Biology, Metastasis Research Laboratory, The University of Texas MD Anderson Cancer Center, 1515 Holcombe Boulevard, Unit 173, Houston, TX 77030, USA

E-mail: ifidler@mdanderson.org

Key words: microarray, epithelial-mesenchymal transition, cell culture, media formulation

\section{Introduction}

Recent reports have sought to raise awareness of the growing number of cancer research studies whose findings cannot be independently reproduced (1). Indeed, investigators from the Hematology and Oncology Department at Amgen in Thousand Oaks, CA, USA, were only able to confirm the scientific results in six out of $53(11 \%)$ reports that were regarded as landmark studies (1). A similar assessment of 67 projects (47 of which were oncology studies) by researchers from Bayer HealthCare (Leverkusen, Germany) revealed that only one-quarter of the published data could be reproduced (2). Several reasons have been proposed to explain the high rate of contrasting results among different laboratories, including investigator bias, inappropriate statistical analysis of results, and insufficient sample size $(1,2)$. Investigator bias is a broad category that includes manipulation of the analysis and selective reporting of data (3). It is also well known that the smaller the experimental sample size, the less likely the research findings are to be true (3). Alterations in cell culture conditions are also reported to skew experimental results and increase the likelihood that a study cannot be replicated (4). However, there are no comprehensive analyses of the effects of cell culture modifications on the cancer cell transcriptome.

Cancer cell lines are an indispensable component of a translational research program and have played a critical role in several important discoveries, including identification of $B R A F$ mutations in human tumors (5), development of targeted therapeutic agents (6), determining mechanisms of therapeutic resistance (7), and many others (8). The extent that investigators rely on cancer cell lines for their studies is exemplified by the current collection of 200 lung cancer cell lines, which have been the subject of $>9,000$ citations (9). These and other cancer cell lines are maintained in defined media that are isosmotic and contain a buffer, inorganic salts, nutrients (amino acids and vitamins) and an energy source (usually glucose) to permit normal cell metabolism. However, the composition of media formulations can vary widely. For example, complete Eagle's minimum essential medium (MEM) contains $1,000 \mathrm{mg} / \mathrm{l}$ of glucose, whereas the concentration of glucose in Dulbecco's modified Eagle's medium (DMEM) containing the high glucose modification is $4,500 \mathrm{mg} / \mathrm{l}$. The concentration of glucose present in Roswell Park Memorial Institute (RPMI)-1640 medium falls between MEM and DMEM and is 2,000 mg/l. 
It is widely known that the tumor microenvironment has a profound impact on determining the gene expression patterns of cancer cells (10). Cancer cells may also influence gene expression of normal (non-transformed) cell populations residing in the tumor microenvironment and the extent of the gene modulation occurring in both compartments may be quantitatively assessed experimentally using cross-species hybridization of microarrays (11). Here, we varied the in vitro microenvironment of MDA-MB-231 breast cancer cells by adjusting their cell culture conditions and then constructed gene expression profiles on the cells to determine the possibility that cell culture modifications could contribute to the inability to reproduce experimental results. The resulting data emphasize that in order to obtain reproducible results for cancer cells grown in culture, one must adhere to the precise details regarding media formulation, supplemental nutrition, and the density of the cell preparation at the time of analysis.

\section{Materials and methods}

Antibodies. The following antibodies were used in this study: anti-IL-8, anti-E-cadherin (Invitrogen Life Technologies, Carlsbad, CA, USA); anti-S100A4, anti-VIM, anti-CD44 (Cell Signaling Technology, Inc., Beverly, MA, USA); anti-CD24 (R\&D Systems, Minneapolis, MN, USA); anti- $\beta$-actin (AC-15) (Sigma-Aldrich, St. Louis, MO, USA); goat anti-mouse IgGhorseradish peroxidase (HRP), goat anti-rabbit IgG-HRP (Santa Cruz Biotechnology, Inc., Santa Cruz, CA, USA).

Cell lines and cell culture conditions. Human MDA-MB-231 breast cancer cells (12) were maintained as a monolayer culture in MEM, DMEM, or RPMI-1640 supplemented with L-glutamine, sodium pyruvate, non-essential amino acids, a 2-fold vitamin solution, and penicillin-streptomycin (Invitrogen Life Technologies). Fetal bovine serum (FBS) (HyClone, Logan, UT, USA) or horse serum (HS) (Invitrogen Life Technologies) was added to the media. All tissue culture reagents were free of endotoxin as determined by the Limulus Amebocyte Lysate assay (Associates of Cape Cod, Inc., Woods Hole, MA, USA). MDA-MB-231 cells were free of the following murine pathogens: Mycoplasma species, Hanta virus, hepatitis virus, minute virus, adenovirus (MAD1, MAD2), cytomegalovirus, ectromelia virus, lactate dehydrogenase-elevating virus, polyma virus, and Sendai virus (assayed by the Research Animal Diagnostic Laboratory, University of Missouri, Columbia, MO, USA). MDA-MB-231 breast cancer cells were tested at the MD Anderson Characterized Cell Line Core Facility using short tandem repeats DNA profiling.

Microarray analysis. Total RNA was extracted from the cultured cells by using the mirVana miRNA Isolation kit (Life Technologies, Grand Island, NY, USA) according to the manufacturer's instructions. The integrity of the RNA fraction was determined using a Bio-Rad Experion Bioanalyzer (Bio-Rad, Hercules, CA, USA) as a surrogate for mRNA quality control. Biotin-labeled cRNA samples were prepared by using the Illumina Total Prep RNA Amplification kit and $1.5 \mu \mathrm{g}$ of biotinylated cRNA sample was hybridized to HumanHT-12 v4.0 Expression BeadChip (Illumina, Inc., San Diego, CA, USA). BeadChips were scanned with an Illumina BeadArray Reader and the microarray data were normalized using the quantile normalization method in the Linear Models for Microarray Data package in the R language environment (12). All statistical analyses were performed using the BRB-ArrayTools software program (version 4.0) (13).

Western blot analysis. Western blot analysis was used to confirm the results of the microarray data. MDA-MB-231 cancer cells $\left(2 \times 10^{6}\right.$ cells $)$ were plated onto $100 \mathrm{~mm}$ culture dishes and maintained in the various media formulations containing different concentrations (or types) of sera. Whole-cell lysates of cancer cells were obtained when cancer cells reached the appropriate experimental cell density by lysing cells in buffer [10 mM Tris ( $\mathrm{pH} 8.0), 1 \mathrm{mM}$ EDTA, $0.1 \%$ SDS, $1 \%$ deoxycholate, $1 \%$ NP40, $0.14 \mathrm{M} \mathrm{NaCl}, 1 \mu \mathrm{g} / \mathrm{ml}$ leupeptin, $1 \mu \mathrm{g} / \mathrm{ml}$ aprotinin, and $1 \mu \mathrm{g} / \mathrm{ml}$ pepstatin] containing a protease inhibitor mixture (Roche Diagnostics, Indianapolis, IN, USA) (12). Next, $30 \mu \mathrm{g}$ of total protein was separated by electrophoresis on 4-12\% Nu-PAGE gels (Life Technologies) and transferred to nitrocellulose membranes. Membranes were blocked for $1 \mathrm{~h}$ and then incubated overnight at $4{ }^{\circ} \mathrm{C}$ with primary antibodies $(1: 1,000)$. The membranes were rinsed, incubated with HRP-conjugated secondary antibodies (1:3,000), and visualized by enhanced chemiluminescence (Amersham Pharmacia Biotech, Piscataway, NJ, USA). To ensure equal protein loading, the blots were stripped and reprobed with an anti- $\beta$-actin antibody (Sigma-Aldrich). Quantification of protein levels in the western blots was performed using ImageJ software (National Institutes of Health, Bethesda, MD, USA).

Real-time reverse transcription polymerase chain reaction. Microarray results for interleukin-8 (IL-8), S100A4, vimentin $(V I M)$, E-cadherin $(C D H 1), C D 44$, and $C D 24$ were validated using real-time reverse transcription polymerase chain reaction (RT-PCR). Total RNA was extracted from the MDA-MB-231 cancer cells using the Qiagen RNeasy Mini kit (Qiagen, Valencia, CA, USA) according to the manufacturer's instructions. First-strand cDNA was synthesized from $5 \mu \mathrm{g}$ RNA using SuperScript III Reverse Transcriptase (Invitrogen Life Technologies). RT-PCR was performed using TaqMan ${ }^{\circledR}$ Universal PCR Master Mix and quantified with Applied Biosystems 7500 Real-Time PCR system (Applied Biosystems, Foster City, CA, USA). The following TaqMan ${ }^{\circledR}$ Gene Expression assays were used in our validation study; human IL-8 (Hs00174103-ml); human SlOOA4 (Hs00243202_m1); human VIM (Hs00185584_m1); human CDH1 (Hs01023894_m1); human CD44 (Hs01075861_m1) and human CD24 (Hs02379687_s1) (all from Applied Biosystems). $18 \mathrm{~S}$ rRNA was used as an endogenous control. Relative mRNA expression in the cells was calculated using the $\Delta \Delta \mathrm{Ct}$ method (14) and the results are expressed as the mean \pm standard deviation (SD) of mRNA relative to that of control.

Statistical analysis. All statistical analyses were performed using BRB-ArrayTools version 4.3.2 under the R language environment. The microarray data were normalized using the quantile normalization method in the Linear Models for Microarray Data package. A two-sample t-test was applied to gene expression data from three groups of samples and expression of genes and a $\mathrm{P}<0.001$ was considered statistically 

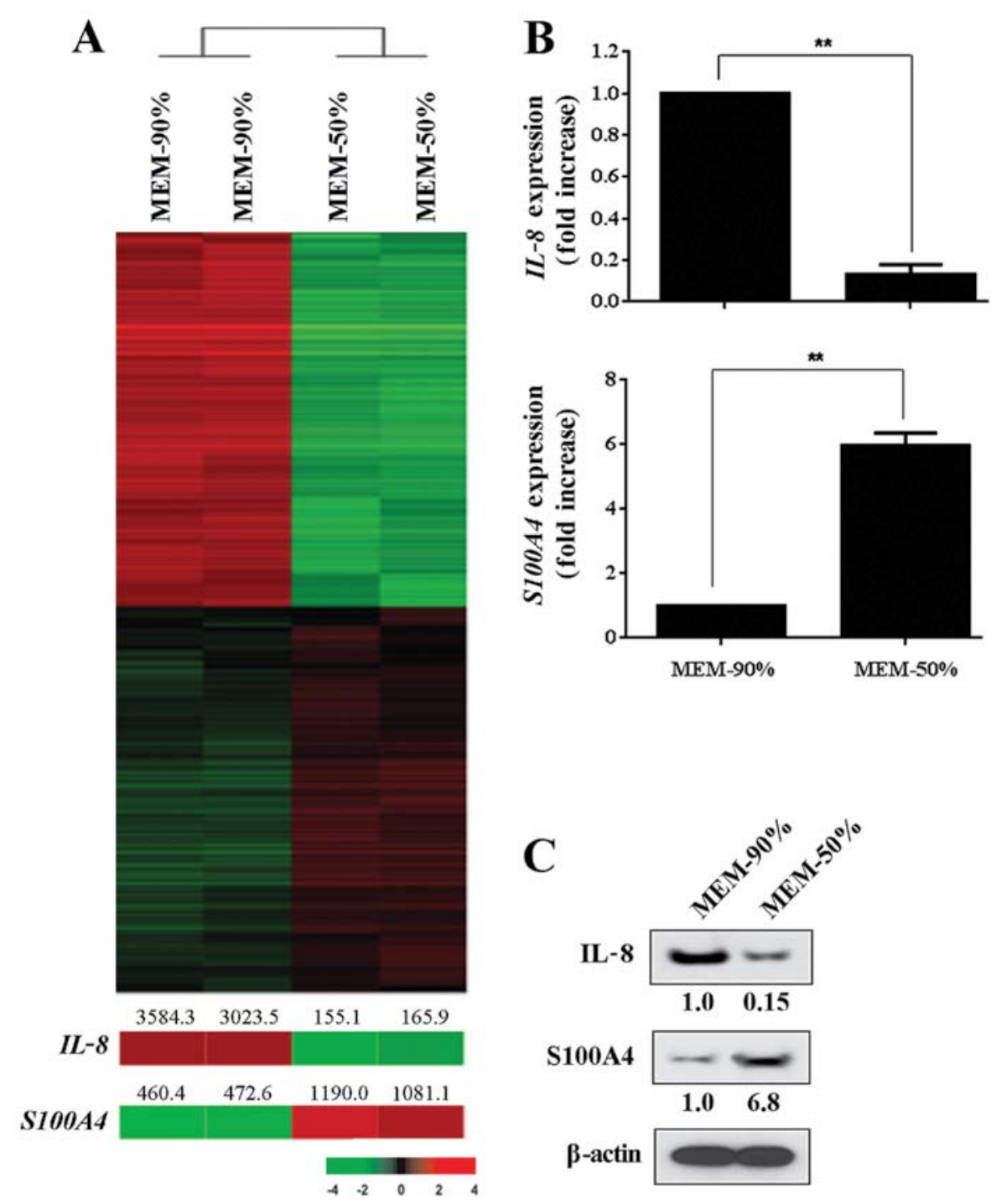

Figure 1. Differential gene expression in confluent (90\%) and subconfluent (50\%) MDA-MB-231 breast cancer cells. (A) Hierarchical clustering analysis of confluent and subconfluent MDA-MB-231 breast cancer cells. Genes with an expression level exceeding a 2-fold difference relative to the median value were selected for hierarchical clustering analysis ( $\mathrm{n}=2,234$ genes). The data are presented in matrix format in which rows represent individual genes and columns represent each culture condition. Each cell in the matrix represents the expression level of a gene feature in an individual culture. The color red or green in cells reflects relative high or low expression levels, respectively, as indicated in the scale bar (log2-transformed scale). (B) Real-time reverse transcription polymerase chain reaction (RT-PCR) validation of interleukin-8 $(I L-8)$ and S100A4 expression using RT-PCR and (C) confirmation of IL-8 and S100A4 protein with western blot analysis. Values shown are means \pm standard deviation (SD) from three independent experiments. " $\mathrm{P}<0.01$.

significant. This stringent significance threshold was used to limit the number of false-positive findings. We also performed a global test of whether the expression profiles differed between the classes by permuting the labels of which arrays corresponded to which classes. For each permutation, the P-values were recomputed and the number of genes with significant expression levels of $<0.001$ was noted. Cluster analyses were performed with the Cluster software program and heat maps were generated using the TreeView software program (15).

Accession numbers. The microarray data have been deposited in the Gene Expression Omnibus under accession number GSE61670.

\section{Results}

Cell density affects patterns of gene expression in $M D A-M B-231$ cancer cells. To begin to study the effects of the cell culture environment on the cancer cell transcriptome, we first examined how alterations in cell density affect patterns of gene expression of MDA-MB-231 breast cancer cells. The cells were grown as monolayers in MEM supplemented with $10 \%$ FBS and harvested for analysis when they were 50 or $90 \%$ confluent in culture dishes. We noted that this alteration in cell density resulted in the differential expression of 2,234 genes (Fig. 1A). Specifically, 1,100 genes were significantly upregulated in cancer cells that were $90 \%$ confluent when compared to cells that were $50 \%$ confluent. A similar number of genes (1,134 genes) were significantly downregulated in $90 \%$ confluent cancer cells. We selected $I L-8$ and S100A4 from the upregulated and downregulated gene sets for validation using RT-PCR, because these two genes were among the most differentially expressed in their corresponding gene sets. The expression of $I L-8$ mRNA in cells that were $90 \%$ confluent in MEM was 9-fold greater than that of cells cultured to $50 \%$ confluence in the same medium. In contrast, we noted a 6-fold downregulation in S100A4 mRNA 

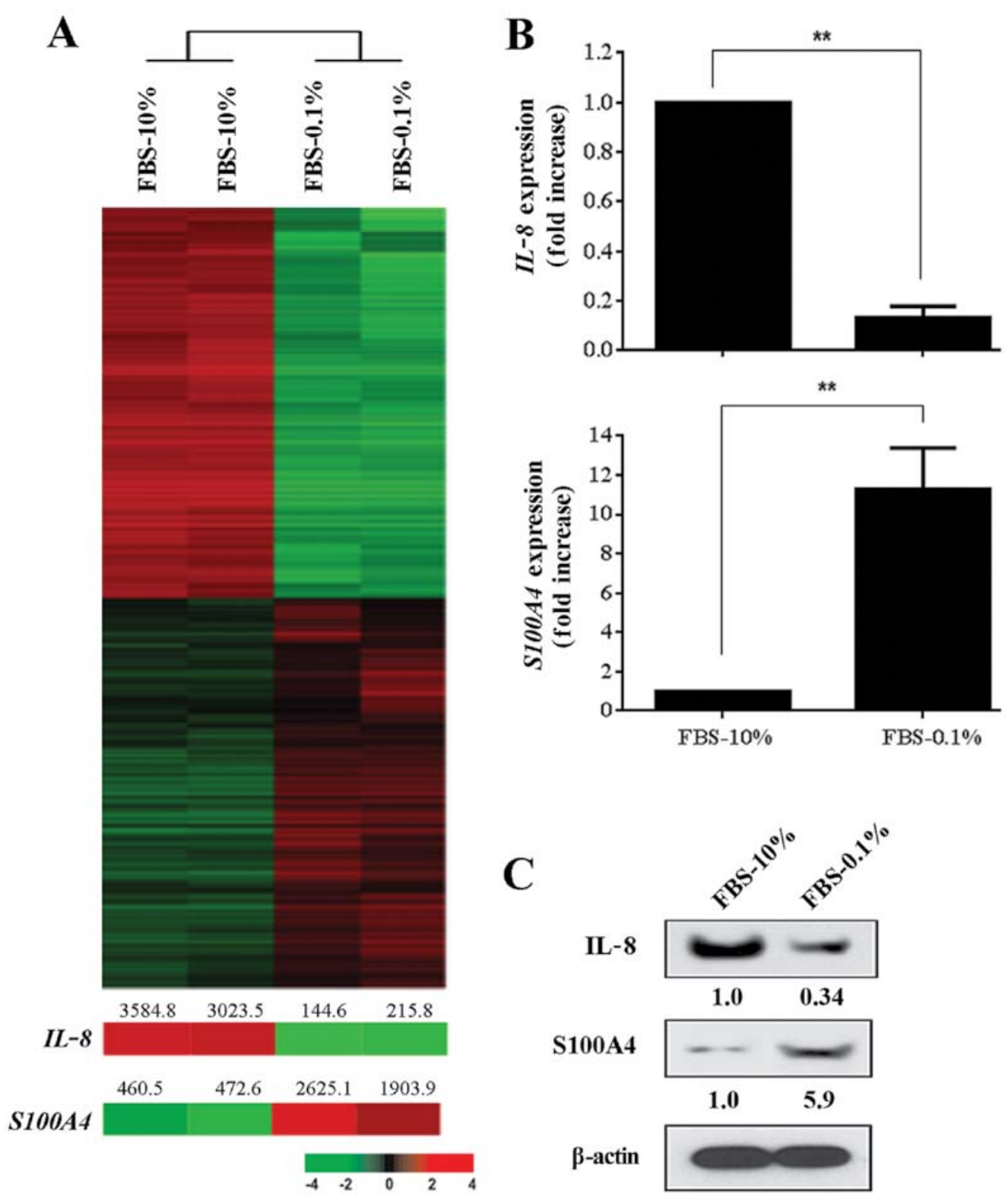

Figure 2. Differential gene expression between MDA-MB-231 breast cancer cells cultured in minimum essential medium (MEM) supplemented with 10 and $0.1 \%$ fetal bovine serum (FBS). (A) Hierarchical clustering analysis of cells maintained in MEM containing 10\% FBS and MEM containing 0.1\% FBS. Genes with an expression level exceeding a 2-fold difference relative to the median value were selected for hierarchical clustering analysis ( $\mathrm{n}=2,981 \mathrm{genes}$ ). The data are presented in matrix format as described in Fig. 1. (B) Real-time reverse transcription polymerase chain reaction (RT-PCR) analysis of interleukin-8 (IL-8) and S100A4 expression. (C) Western blot analysis of IL-8 and S100A4. Values shown are means \pm standard deviation (SD) from three independent experiments. ${ }^{* *} \mathrm{P}<0.01$.

expression in cells that were $90 \%$ confluent in comparison to cells that were analyzed once they reached $50 \%$ confluence. We confirmed the differential expression of $I L-8$ and S100A4 at the protein level using western blot analysis (Fig. 1C).

Concentration of FBS affects MDA-MB-231 cancer cell gene expression. Next, we analyzed how varying the concentration of FBS affected gene expression in MDA-MB-231 breast cancer cells. The concentration of FBS in several same passage cultures of MDA-MB-231 cancer cells was adjusted to either 0.1 or $10 \%$ and all cells were analyzed when cultures reached $90 \%$ confluency. We found almost 3,000 genes that were differentially expressed between MDA-MB-231 cells cultured in $10 \%$ FBS and those cultured in 0.1\% FBS (Fig. 2A). A total of 1,489 genes were expressed at significantly higher levels in cells cultured in 10\% FBS when compared to cells cultured in $0.1 \%$. Once again, $I L-8$ and $S 100 A 4$ gene expressions were among the more differentially regulated genes and both were examined in greater detail. RT-PCR analysis revealed that $I L-8$ was upregulated by 10 -fold in MDA-MB-231 cancer cells that were grown in $10 \%$ FBS, whereas S100A4 was downregulated by 10-fold in cells maintained in 10\% FBS (Fig. 2B). We confirmed these findings by western blot analysis, which showed a 3-fold increase of IL-8 expression in MDA-MB-231 cancer cells grown in $10 \%$ FBS and almost a 6 -fold increase in $\mathrm{S} 100 \mathrm{~A} 4$ protein expression when cells are grown in the reduced concentration (0.1\%) of FBS (Fig. 2C).

Virtually any type of animal is capable of serving as a donor for serum, but some animal sera are used more often than others. Fetal bovine serum (FBS) and horse serum 
$\mathbf{A}$
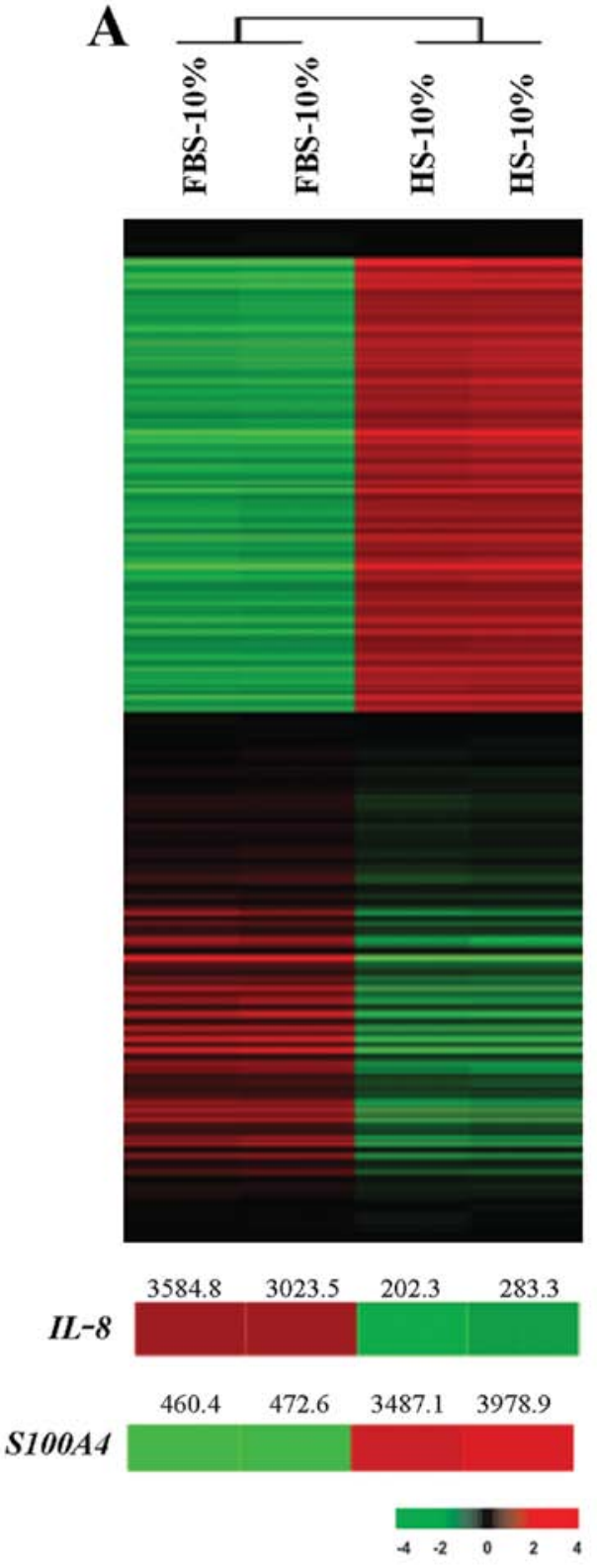

B
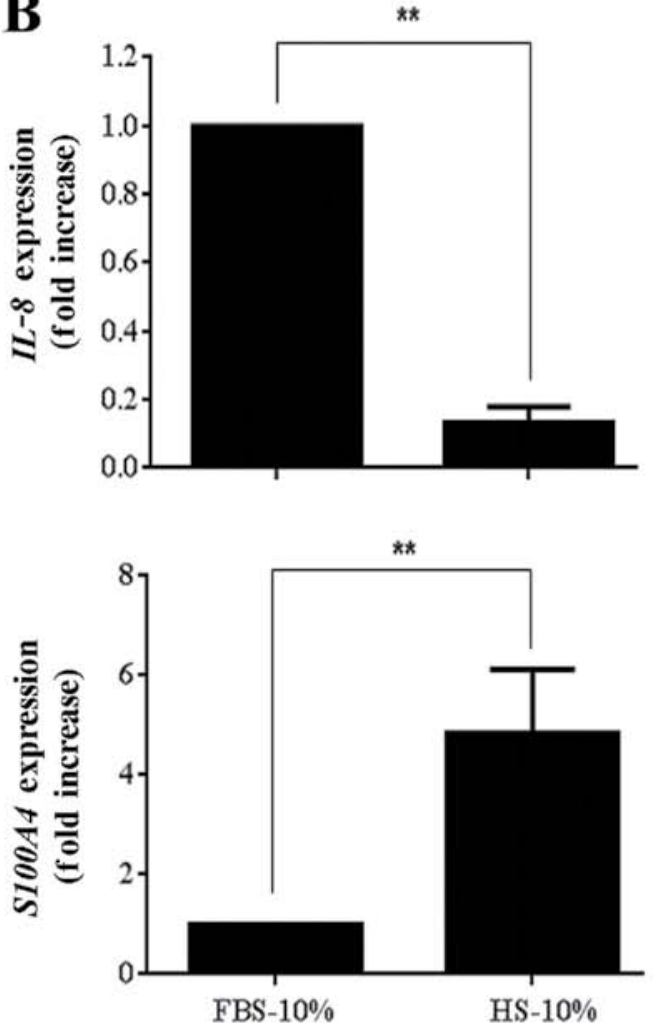

C

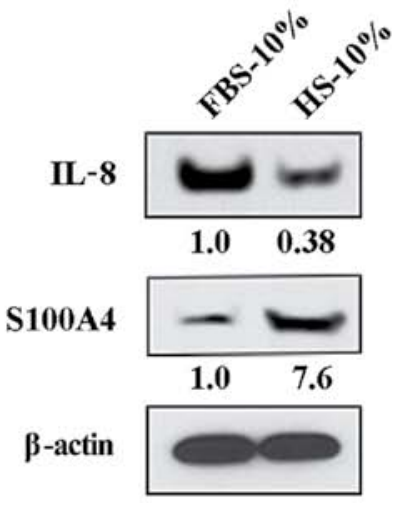

Figure 3. Differential gene expression between MDA-MB-231 breast cancer cells cultured in minimum essential medium (MEM) supplemented with $10 \%$ fetal bovine serum (FBS) and cells cultured in MEM containing 10\% horse serum (HS). (A) Hierarchical clustering analysis of cells maintained in 10\% FBS and $10 \%$ HS. Genes with an expression level exceeding a 2-fold difference relative to the median value were selected for hierarchical clustering analysis ( $\mathrm{n}=2,981 \mathrm{genes)}$. (B) Real-time reverse transcription polymerase chain reaction (RT-PCR) analysis of interleukin-8 (IL-8) and S100A4. (C) Western blot analysis of IL-8 and S100A4. Values shown are means \pm standard deviation (SD) from three independent experiments. ${ }^{* *} \mathrm{P}<0.01$.

(HS) are among the more common types of sera used for culturing mammalian cells and we evaluated the effects of these sera on MDA-MB-231 cancer cell gene expression. In this series of experiments, we maintained the density of cell cultures at the time of analysis constant at $90 \%$. While altering the source of the sera used in cell culture significantly affected cancer cell gene expression, the overall effect was less than that observed when we modified the concentration of FBS in the culture media or when we analyzed cells grown at different cell densities. We recorded a total of 422 differentially expressed genes in a comparison between MDA-MB-231 cancer cells grown in MEM containing $10 \%$ FBS and those grown in MEM containing $10 \% \mathrm{HS}$. A total of 235 and 187 genes were significantly upregulated and down- regulated, respectively, in cancer cells in the $10 \%$ FBS cell group (Fig. 3A). IL-8 and S100A4 were among the differently modulated genes and expression levels were confirmed using RT-PCR (Fig. 3B) and western blot analysis (Fig. 3C). IL-8 protein expression in MDA-MB-231 cancer cells grown in media supplemented with $10 \%$ FBS was $\sim 3$-fold higher than of cells maintained in $10 \%$ HS. In contrast, S100A4 protein expression was negligible in MEM containing FBS, but was markedly upregulated in media containing HS.

Cell culture media formulations exert the most profound effect on cancer cell gene expression. To determine how different media formulations affect cancer cell gene expression, we cultured MDA-MB-231 breast cancer cells in MEM, DMEM, 
A
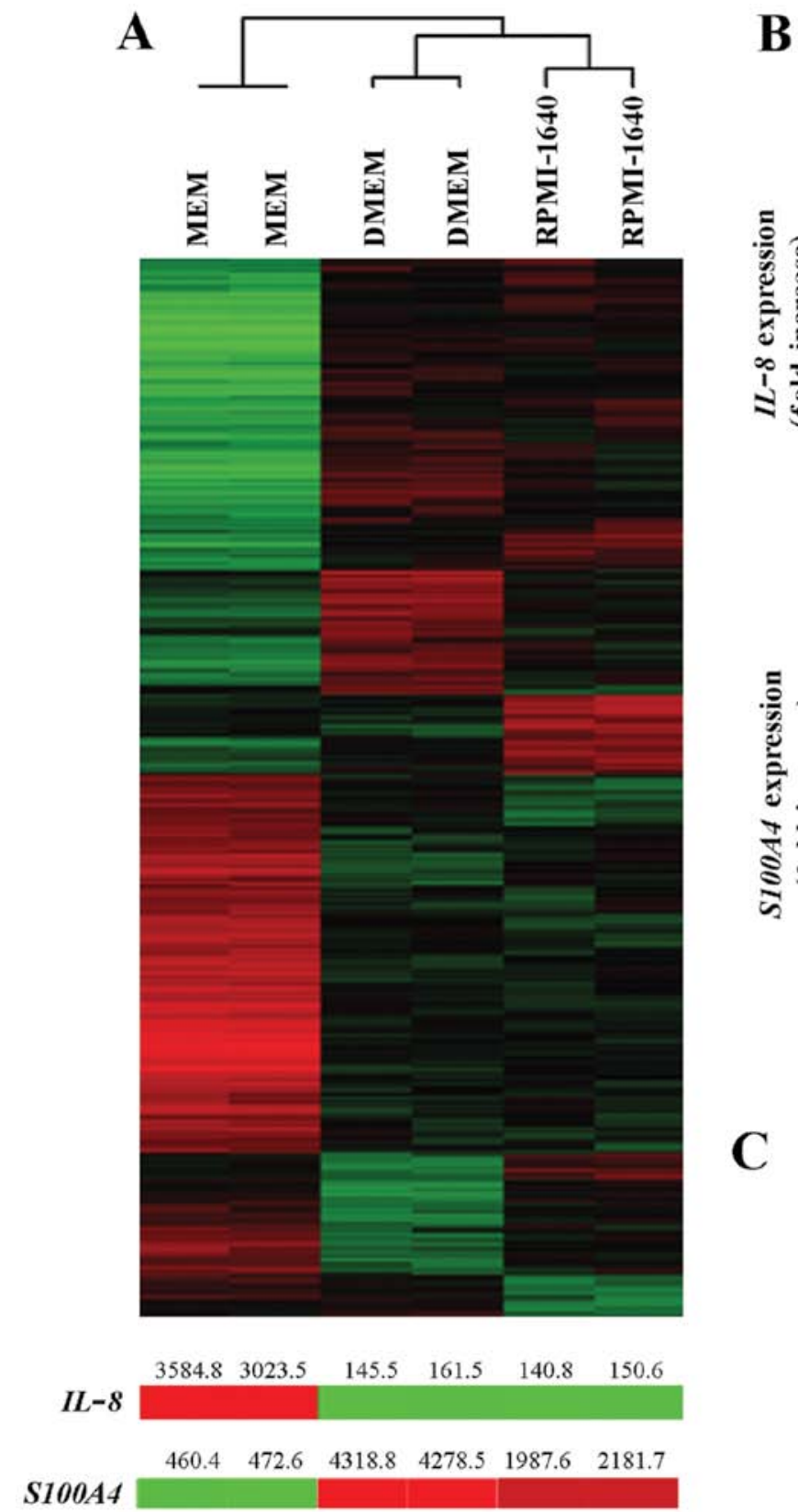

B
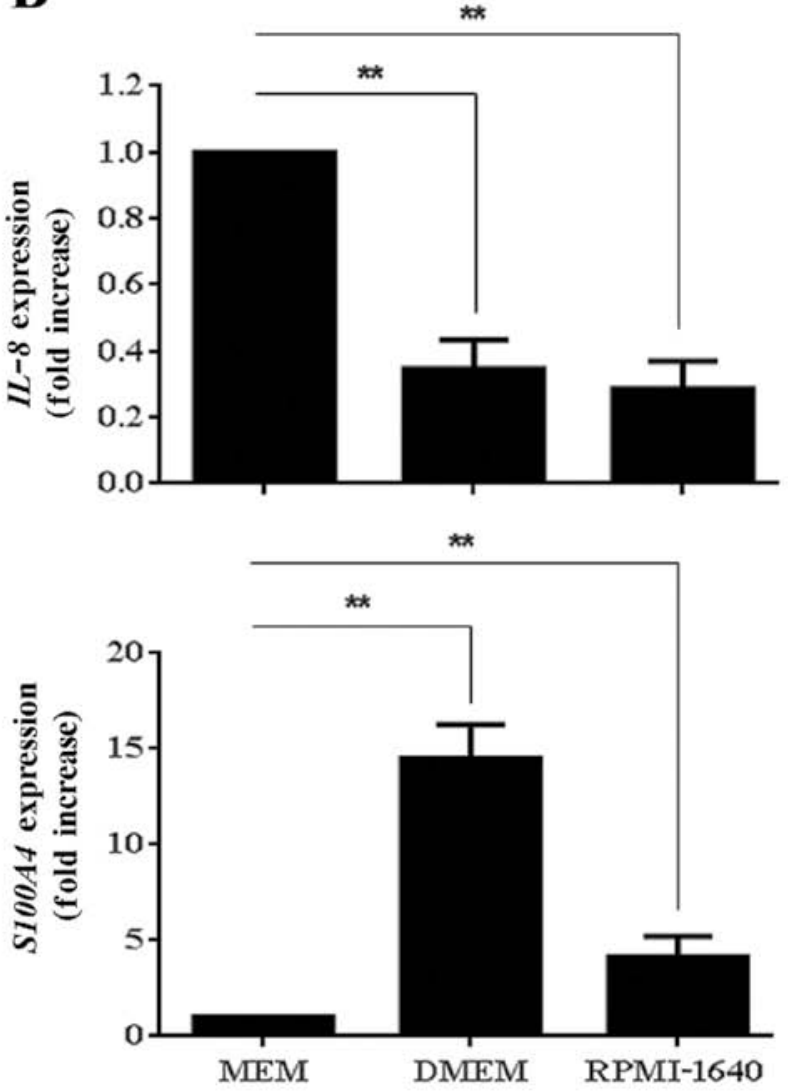

C

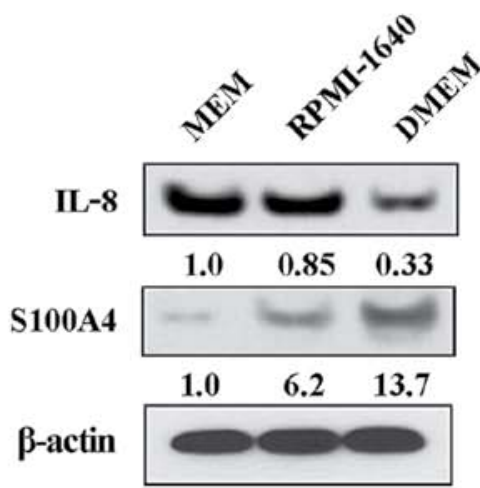

Figure 4. Differential gene expression between MDA-MB-231 breast cancer cells maintained in different media formulations. (A) Hierarchical clustering analysis of MDA-MB-231 breast cancer cells cultured in minimum essential medium (MEM), Dulbecco's modified Eagle's medium (DMEM), and Roswell Park Memorial Institute (RPMI)-1640 medium that were all supplemented with $10 \%$ fetal bovine serum (FBS). (B) Real-time reverse transcription polymerase chain reaction (RT-PCR) analysis of interleukin-8 $(I L-8)$ and S100A4. (C) Protein levels of IL-8 and S100A4 were measured by western blot analysis. Values shown are means \pm standard deviation $(\mathrm{SD})$ from three independent experiments. ${ }^{* *} \mathrm{P}<0.01$.

or RPMI-1640 media, all containing $10 \%$ FBS, and when the cultures reached $90 \%$ confluence, we extracted total RNA from the cells and applied it to beadchip microarrays for analysis. A total of 8,925 genes were differentially expressed when we compared microarray results from MDA-MB-231 cancer cells grown in MEM with those cells grown in DMEM or RPMI-1640 (Fig. 4A). Specifically, 1,409 genes were highly expressed in MEM as compared to DMEM and RPMI-1640; 840 genes were highly expressed in DMEM as compared to MEM and RPMI-1640; and 1,662 genes were highly expressed in RPMI-1640 as compared to MEM and DMEM. The microarray analysis predicted IL- 8 expression levels would be greatest in MDA-MB-231 cells that were grown in MEM and least in cells that were maintained in DMEM and RPMI-1640 and this finding was confirmed by RT-PCR (Fig. 4B). S100A4 gene expression was significantly greater in MDA-MB-231 cells grown in DMEM and RPMI-1640 in comparison to cells grown in MEM and this was also validated by RT-PCR (Fig. 4B). Indeed, S100A4 gene expression levels were 15- and 5-fold greater in cells grown in DMEM and RPMI-1640, respectively, as compared to cells grown in MEM. Western blot analysis of IL-8 and S100A4 paralleled the gene expression results (Fig. 4C).

Cell culture media formulations modulate expression of genes associated with EMT. Data mining on the expression array sets suggested that genes frequently associated with 

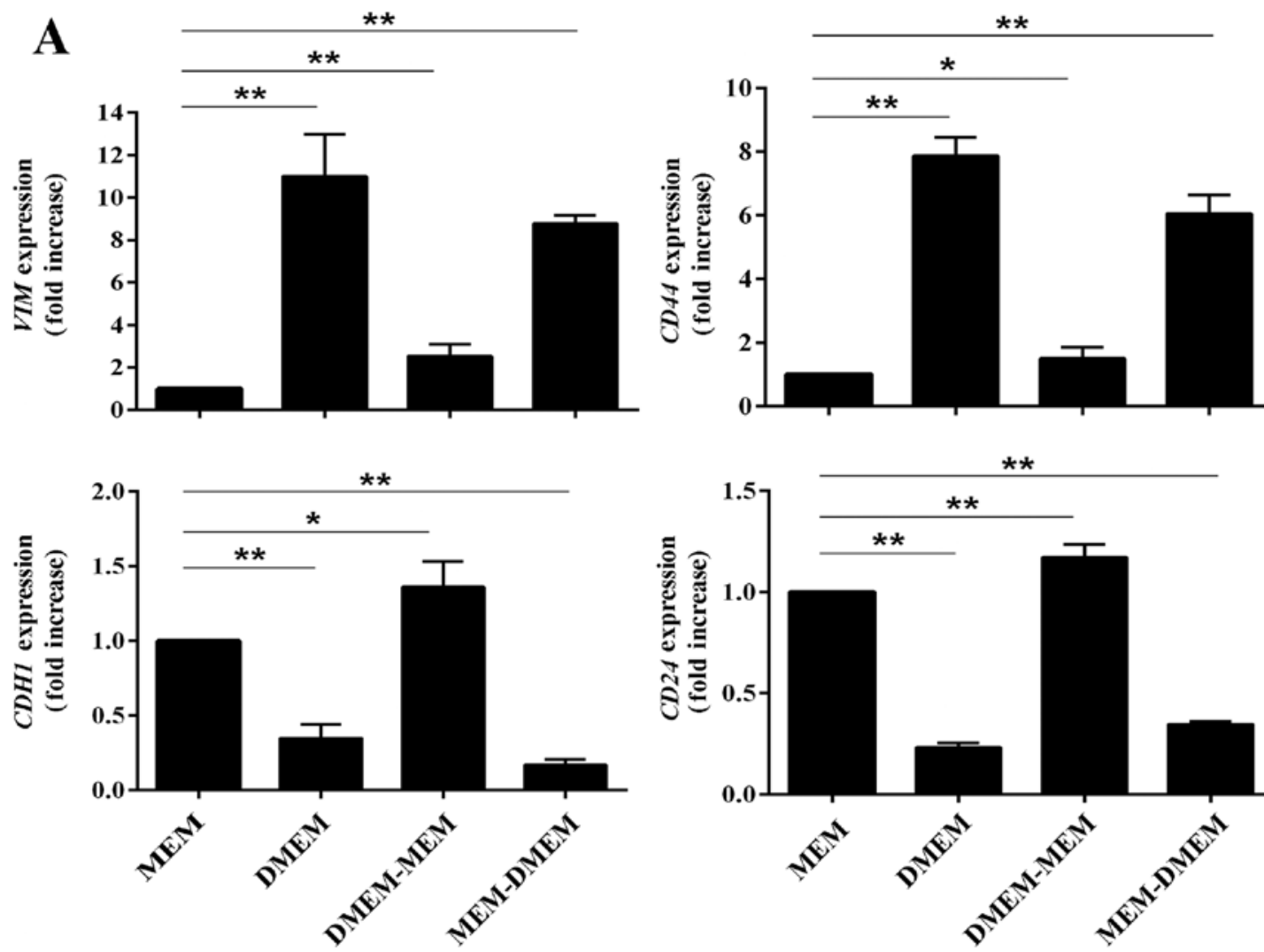

B

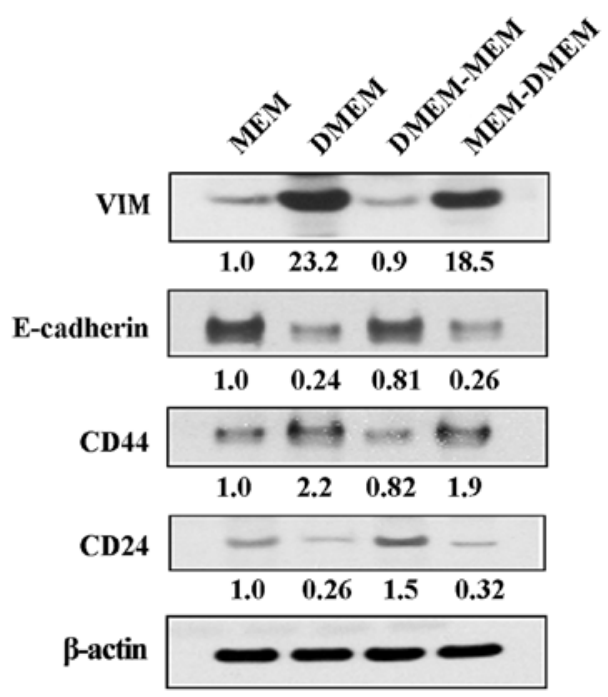

Figure 5. Expression of epithelial-mesenchymal transition (EMT) markers in MDA-MB-231 breast cancer cells cultured in minimum essential medium (MEM) and Dulbecco's modified Eagle's medium (DMEM) formulations. (A) MDA-MB-231 breast cancer cells were cultured in MEM or DMEM containing 10\% fetal bovine serum (FBS) for $48 \mathrm{~h}$. Real-time reverse transcription polymerase chain reaction (RT-PCR) analysis of vimentin (VIM), CDH1, CD44, and CD24 gene expression. To determine if changes in gene expression were reversible, the culture media was changed from MEM to DMEM (or from DMEM to MEM) and the cells were analyzed $48 \mathrm{~h}$ later. Fold-increase refers to the ratio of mRNA levels relative to that of cells cultured in MEM. (B) Western blot analysis comparing protein expression of VIM, E-cadherin, CD44, and CD24. ${ }^{*} \mathrm{P}<0.05,{ }^{* *} \mathrm{P}<0.01$.

epithelial-mesenchymal transition (EMT) were differentially modulated by the various media formulations. MDA-MB-231 cancer cells that were grown in MEM had a tendency to express significantly higher expression levels of epithelial cell markers E-cadherin $(C D H I)$ and $C D 24$, whereas the same cells grown in DMEM expressed higher levels of mesenchymal markers $V I M$ and $C D 44$. We confirmed the gene expression array using RT-PCR analysis. Gene expression levels of VIM and CD44 in MDA-MB-231 cancer cells were dramatically suppressed when the cells were grown in MEM in comparison to cells grown in DMEM (Fig. 5A, upper panel). VIM and CD44 expression levels were at least 8-fold higher in cells that were maintained in DMEM. However, expression levels of these two genes could be easily modulated by simply switching the cells to a different media formulation for a period of $48 \mathrm{~h}$. When DMEM was replaced by MEM, expression levels of CD44 and VIM dropped dramatically; when MEM was replaced by DMEM expression, levels of CD44 and VIM increased. 
The gene expression patterns of $C D H 1$ and $C D 24$ were diametrically opposed to that of $C D 44$ and VIM. That is, $C D H I$ and $C D 24$ gene expression were elevated in MDA-MB-231 cancer cells when they were grown in MEM and both were suppressed when cells were maintained in DMEM (Fig. 5A, lower panel). Similar to our analysis of CD44 and VIM, gene expression of $C D H 1$ and $C D 24$ was plastic and influenced by the in vitro microenvironment. When the MDA-MB-231 cancer cell media was changed from MEM to DMEM for $48 \mathrm{~h}$, expression levels of $C D H 1$ and $C D 24$ were significantly reduced. All of the gene expression results were validated at the protein level by western blot analysis (Fig. 5B).

\section{Discussion}

Cancer cell lines play an invaluable role in the drug discovery process (16) and in identifying molecular mechanisms of therapeutic resistance (7). Because tumor cell lines consist of pure populations of cancer cells, they are frequently more advantageous for study as compared to tumor tissue (8). For example, cancer cell lines provide a source of high quality DNA, RNA, and proteins that may facilitate testing and data interpretation (8). However, the misidentification of cell lines and their cross-contamination have led to a number of misleading and erroneous publications in the scientific literature $(17,18)$. Recently, several granting agencies and scientific journals have sought to handle this problem by requiring investigators to provide cell line authentication for human cancer cells using short tandem repeat profiling (18). In the present report, we provide convincing evidence that the replication of results on cultured cancer cells also requires rigid adherence to the cell culture formulation used to maintain cells and reporting of the cell density reached at the time of analysis.

One of the more striking observations of our study was that in our whole-genome microarray analysis of MDA-MB-231 breast cancer cells, approximately one-quarter $(25.6 \%)$ of all genes were differentially expressed when we examined cells that were grown in different media formulations. While we had predicted that several genes would be differentially modulated by the various media preparations, we did not anticipate the extent of differential gene expression observed in our study. Genes associated with the EMT program were upregulated or downregulated simply by switching the cells to a different media formulation (different concentration of glucose) for a period of $48 \mathrm{~h}$. EMT plays a critical role during development and is also observed in the process of tissue repair (19). Invading and metastasizing carcinoma cells revive the EMT program by upregulating mesenchymal proteins and by suppressing expression of epithelial proteins (20). EMT has been the focus of much recent investigation because cancer cells undergoing EMT have been shown to obtain stem cell-like properties and become resistant to anticancer agents (21). Our results demonstrate that EMT gene and protein expression is remarkably plastic in cultured cancer cells and that EMT is highly influenced by the tissue culture microenvironment. It is tempting to speculate that the elevated glucose concentration present in DMEM and RPMI-1640 relative to that found in MEM was responsible for the induction of the EMT program in MDA-MB-231 breast cancer cells. Evidence to support this contention comes from studies of renal tubular cells (22) and peritoneal mesothelial cells (23), which activate the EMT program in response to stimulation with high concentrations of glucose. Moreover, a recent study demonstrated that the small fraction of cancer cells with stem-like properties that exists in a tumor could be dramatically increased in the cell culture environment by culturing the cells in high concentrations of glucose (24). These investigations lend credibility to the experimental approach used in our study and suggest that additional study in this area is warranted.

Two genes that have been linked to breast cancer progression, $I L-8$ and $S 100 A 4$, were differentially modulated by every cell culture modification that we examined. S100A4 is a small calcium-binding protein that has been shown to promote migration, invasion, and anchorage-independent growth of breast cancer cells (25). In the present report, S100A4 was upregulated in MDA-MB-231 cancer cells under conditions of low cell density, minimal FBS supplementation, HS, and when cells are grown in a DMEM formulation. Alternatively, S100A4 expression was negligible when cells were maintained in media supplemented with $10 \%$ FBS and when cells achieved a confluent state. IL- 8 is a human chemokine that is produced by a variety of different cell types. In healthy tissues, IL- 8 is minimally expressed, but can be rapidly induced 100-fold in response to pro-inflammatory cytokines, such as tumor necrosis factor, IL-1, bacterial or viral products, and cellular stress (26). IL-8 contributes to the progression of several types of tumors by mediating cancer cell migration and stimulating tumor neovascularization (27). More recent studies have shown that IL-8 signaling may function as a key factor in the regulation of breast cancer stem cell activity (28). In our study, IL-8 expression was enriched in confluent MDA-MB-231 breast cancer cells that were maintained in MEM containing 10\% FBS.

Collectively, our results show that the gene expression patterns of cancer cells can vary significantly according to the conditions under which they are cultured. While the introduction of measures that ensure the identification of cancer cell lines used in research investigations will undoubtedly improve the reproducibility of translational oncology research, accurate documentation of cell culture conditions is essential for replicating results from in vitro studies of cancer cells.

\section{Acknowledgements}

This study was supported in part by funds from Cancer Center Support Core grant CA16672 and grant 2RO1-CA154710 from the National Cancer Institute, National Institutes of Health (Bethesda, MD, USA).

\section{References}

1. Begley CG and Ellis LM: Drug development: raise standards for preclinical cancer research. Nature 483: 531-533, 2012.

2. Prinz F, Schlange T and Asadullah K: Believe it or not: how much can we rely on published data on potential drug targets? Nat Rev Drug Discov 10: 712, 2011.

3. Ioannidis JP: Why most published research findings are false. PLoS Med 2: e124, 2005.

4. Bissell M: Reproducibility: the risks of the replication drive. Nature 503: 333-334, 2013.

5. Davies H, Bignell GR, Cox C, et al: Mutations of the BRAF gene in human cancer. Nature 417: 949-954, 2002.

6. Druker BJ, Tamura S, Buchdunger E, et al: Effects of a selective inhibitor of the Abl tyrosine kinase on the growth of Bcr-Abl positive cells. Nat Med 2: 561-566, 1996. 
7. Engelman JA, Zejnullahu K, Mitsudomi T, et al: MET amplification leads to gefitinib resistance in lung cancer by activating ERBB3 signaling. Science 316: 1039-1043, 2007.

8. Gazdar AF, Gao B and Minna JD: Lung cancer cell lines: useless artifacts or invaluable tools for medical science? Lung Cancer 68: 309-318, 2010

9. Gazdar AF, Girard L, Lockwood WW, Lam WL and Minna JD: Lung cancer cell lines as tools for biomedical discovery and research. J Natl Cancer Inst 102: 1310-1321, 2010.

10. Langley RR and Fidler IJ: The seed and soil hypothesis revisited - the role of tumor-stroma interactions in metastasis to different organs. Int J Cancer 128: 2527-2535, 2011.

11. Cascone T, Herynk MH, Xu L, et al: Upregulated stromal EGFR and vascular remodeling in mouse xenograft models of angiogenesis inhibitor-resistant human lung adenocarcinoma. J Clin Invest 121: 1313-1328, 2011.

12. Kim SJ, Kim JS, Park ES, Lee JS, et al: Astrocytes upregulate survival genes in tumor cells and induce protection from chemotherapy. Neoplasia 13: 286-298, 2011.

13. Simon R, Lam A, Li MC, Ngan M, Menenzes S and Zhao Y: Analysis of gene expression data using BRB-ArrayTools. Cancer Inform 3: 11-17, 2007.

14. Livak KJ and Schmittgen TD: Analysis of relative gene expression data using real-time quantitative PCR and the 2(-Delta Delta C(T)) method. Methods 25: 402-408, 2001.

15. Eisen MB, Spellman PT, Brown PO and Botstein D: Cluster analysis and display of genome-wide expression patterns. Proc Nat Acad Sci USA 95: 14863-14868, 1998.

16. Druker BJ and Lydon NB: Lessons learned from the development of an abl tyrosine kinase inhibitor for chronic myelogenous leukemia. J Clin Invest 105: 3-7, 2000.

17. American Type Culture Collection Standards Development Organization Workgroup ASN-0002: Cell line misidentification: the beginning of the end. Nat Rev Cancer 10: 441-448, 2010.
18. Barallon R, Bauer SR, Butler J, et al: Recommendation of short tandem repeat profiling for authenticating human cell lines, stem cells, and tissues. In Vitro Cell Dev Biol Anim 46: 727-732, 2010.

19. Thiery JP, Acloque H, Huang RY and Nieto MA: Epithelial-mesenchymal transitions in development and disease. Cell 139: 871-890, 2009.

20. Tam WL and Weinberg RA: The epigenetics of epithelialmesenchymal plasticity in cancer. Nat Med 19: 1438-1449, 2013.

21. Scheel C and Weinberg RA: Cancer stem cells and epithelial-mesenchymal transition: concepts and molecular links. Semin Cancer Biol 22: 396-403, 2012.

22. Tang L, Li H, Gou R, Cheng G, Guo Y, Fang Y and Chen F: Endothelin-1 mediated high glucose-induced epithelial-mesenchymal transition in renal tubular cells. Diabetes Res Clin Pract 104: 176-182, 2014

23. Yu MA, Shin KS, Kim JH, et al: HGF and BMP-7 ameliorate high glucose-induced epithelial-to-mesenchymal transition of peritoneal mesothelium. J Am Soc Nephrol 20: 567-581, 2009.

24. Liu PP, Liao J, Tang ZJ, et al: Metabolic regulation of cancer cell side population by glucose through activation of the Akt pathway. Cell Death Differ 21: 124-135, 2014.

25. Kim TH, Kim HI, Soung YH, Shaw LA and Chung J: Integrin (alpha6beta4) signals through Src to increase expression of S100A4, a metastasis-promoting factor: implications for cancer cell invasion. Mol Cancer Res 7: 1605-1612, 2009.

26. Hoffmann E, Dittrich-Breiholz O, Holtmann H and Kracht M: Multiple control of interleukin-8 gene expression. J Leukoc Biol 72: 847-855, 2002.

27. Waugh DJ and Wilson C: The interleukin- 8 pathway in cancer. Clin Cancer Res 14: 6735-6741, 2008

28. Singh JK, Simões BM, Howell SJ, Farnie G and Clarke RB: Recent advances reveal IL-8 signaling as a potential key to targeting breast cancer stem cells. Breast Cancer Res 15: 210, 2013. 